\title{
Stereotactic radiosurgery combined with immune checkpoint inhibitors or kinase inhibitors for patients with multiple brain metastases of malignant melanoma
}

Stera, Susanne ; Balermpas, Panagiotis ; Blanck, Oliver ; Wolff, Robert ; Wurster, Stefan ; Baumann, Rene ; Szücs, Marcella ; Loutfi-Krauss, Britta ; Wilhelm, Maria-Lisa ; Seifert, Volker ; Rades, Dirk ; Rödel, Claus ; Dunst, Jürgen ; Hildebrandt, Guido ; Arnold, Andreas ; Meissner, Markus ; Kähler, Katharina C

DOI: https://doi.org/10.1097/cmr.0000000000000542

Posted at the Zurich Open Repository and Archive, University of Zurich ZORA URL: https://doi.org/10.5167/uzh-184280

Journal Article

Published Version

Originally published at:

Stera, Susanne; Balermpas, Panagiotis; Blanck, Oliver; Wolff, Robert; Wurster, Stefan; Baumann, Rene; Szücs, Marcella; Loutfi-Krauss, Britta; Wilhelm, Maria-Lisa; Seifert, Volker; Rades, Dirk; Rödel, Claus; Dunst, Jürgen; Hildebrandt, Guido; Arnold, Andreas; Meissner, Markus; Kähler, Katharina C (2019). Stereotactic radiosurgery combined with immune checkpoint inhibitors or kinase inhibitors for patients with multiple brain metastases of malignant melanoma. Melanoma research, 29(2):187-195.

DOI: https://doi.org/10.1097/cmr.0000000000000542 


\title{
Stereotactic radiosurgery combined with immune checkpoint inhibitors or kinase inhibitors for patients with multiple brain metastases of malignant melanoma
}

\author{
Susanne Stera ${ }^{\mathrm{a}, \star}$, Panagiotis Balermpas ${ }^{\mathrm{a}, \mathrm{m}, \mathrm{l}, \mathrm{d}, \star}$, Oliver Blanck ${ }^{\mathrm{m}, \mathrm{e}}$, \\ Robert Wolff $^{\mathrm{m}, \mathrm{c}}$, Stefan Wurster ${ }^{\mathrm{m}, \mathrm{g}}$, Rene Baumann ${ }^{\mathrm{e}, \mathrm{k}}$, Marcella Szücsi, \\ Britta Loutfi-Krauss ${ }^{\mathrm{a}}$, Maria-Lisa Wilhelm ${ }^{\mathrm{m}, \mathrm{i}}$, Volker Seifert ${ }^{\mathrm{c}}$, Dirk Rades ${ }^{j}$, \\ Claus Rödel ${ }^{\mathrm{a}, \mathrm{l}, \mathrm{d}}$, Jürgen Dunst ${ }^{\mathrm{e}}$, Guido Hildebrandt', Andreas Arnold ${ }^{\mathrm{h}}$, \\ Markus Meissner ${ }^{\mathrm{b}, \star}$ and Katharina C. Kähler ${ }^{\mathrm{f}, \star}$
}

The aim was to evaluate toxicity and oncological outcome of combined stereotactic radiosurgery (SRS) and immunotherapy or targeted therapy in patients with multiple brain metastases originating from malignant melanoma. Despite the fact that both SRS and kinase inhibitors or immune checkpoint inhibitors are considered standard treatment options for this indication, the optimal combination and sequence of these modalities remains largely unknown, especially for patients with a high number of brain metastases. For this retrospective analysis, conducted in two large SRS dedicated centers, we identified patients with brain metastases from malignant melanoma and simultaneous application of immunotherapy or targeted therapy within $\mathbf{3 0}$ days of SRS. Forty-eight patients with a total of 250 lesions (median: 3) were treated in 65 single fraction SRS sessions from 2012 to 2018. After a median follow-up of 8.3 months (range: 1.2-43.6 months), the 6-month and 1-year overall survival rates were 75.3 and $50.8 \%$, respectively. The local control rate at one year was $\mathbf{8 9 . 5 \%}$. Immunotherapy and the application of systemic treatment directly before or concomitant to SRS were both associated with improved overall survival $(P=0.037$ and 0.045 , respectively). We observed four grade III toxicities, of which only two can be clearly attributed to the combined treatment. Various combinations of SRS and kinase

\section{Introduction}

Malignant melanoma is one of the most common primary tumors leading to brain dissemination, with $37 \%$ of all patients with melanoma developing brain metastases during the course of their disease [1]. So-called targeted therapies like BRAF and MEK-inhibitors (BRAF/ MEKi), targeting the BRAF-MEK signaling pathway harboring the most common mutation found in melanoma, and recently immune checkpoint inhibitors (ICI) against PD-1 and CTLA-4 for BRAF-wild type tumors or as second-line treatment have become standard options for advanced stage disease and are recommended in clinical guidelines [2,3]. Together with neurosurgical removal, single-fraction stereotactic radiosurgery (SRS) inhibitors or immune checkpoint inhibitors appear feasible and provide promising oncological results and safety profiles for treating few $(n=1-4)$ and also multiple $(n \geq 5)$ melanoma brain metastases. Melanoma Res 29:187-195 Copyright (c) 2018 Wolters Kluwer Health, Inc. All rights reserved.

Melanoma Research 2019, 29:187-195

Keywords: BRAF inhibitor, brain metastases, immunotherapy, melanoma, stereotactic radiosurgery

Departments of ${ }^{a}$ Radiation Oncology, ${ }^{b}$ Dermatology, ${ }^{c}$ Neurosurgery, University Hospital Johann Wolfgang Goethe University, ${ }^{\mathrm{d}}$ German Cancer Consortium (DKTK) Partner Site, Frankfurt, Departments of ${ }^{\mathrm{e}}$ Radiation Oncology,

'Dermatology, University Medical Center Schleswig-Holstein, Kiel, Departments of 'Radiation Oncology, ' $D$ Dermatology, University Medicine Greifswald, Greifswald, 'Department of Radiation Oncology, University Medicine Rostock, Rostock, 'Department of Radiation Oncology, University Medical Center SchleswigHolstein, Lübeck, 'Department of Radiation Oncology, St. Marys Hospital, Siegen, 'German Cancer Research Center (DKFZ), Heidelberg and 'mSaphir Radiosurgery Center, Frankfurt and Northern Germany, Güstrow, Germany

Correspondence to Susanne Stera, MD, Department of Radiation Oncology, University Hospital Johann Wolfgang Goethe University, Theodor Stern Kai 7, 60590 Frankfurt, Germany

Tel: + 49696301 5130; fax: + 49696301 5091; e-mail: susanne.stera@kgu.de

*Susanne Stera, Panagiotis Balermpas, Markus Meissner and Katharina C. Kähler contributed equally to the writing of this article.

Received 29 August 2018 Accepted 9 October 2018

and fractionated stereotactic radiotherapy (SRT) are standard options for 1-4 central nervous system metastases [2], with an emerging role also in the treatment of multiple lesions $(>4)$, with or without the addition of whole-brain radiotherapy (WBRT) [4,5]. Although both SRS/SRT and novel systemic treatments (i.e. targeted therapies and ICI) have proven their efficacy, there is a lack of prospective, randomized data regarding their combination, especially in terms of sequence and toxicity [6]. Moreover, several retrospective analyses indicated a possible improvement in tumor control using these treatments, and also raised concerns regarding toxicity of the combined application [7-10]. This bi-centric study, conducted in two large radiosurgery dedicated centers 
with strict treatment quality control, retrospectively evaluates the oncological results and adverse effects of several combinations of kinase inhibitors or ICI with SRS in a relatively large number of patients $(n=48)$ with one or multiple newly diagnosed and/or recurrent brain metastases (total, $n=250$ ).

\section{Patients and methods Data acquisition}

For this retrospective analysis, we queried the prospectively kept internal databases of our treatment quality harmonized radiosurgery centers and of the according university departments of radiation oncology and dermatological oncology, after institutional review board approval (ethics committee of the Johann Wolfgang Goethe-University Frankfurt, number 395/17, and of the university of Kiel, number B331/17), to identify all patients with one or multiple brain metastases from malignant melanoma, treated with SRS and simultaneous application of immunotherapy or kinase inhibitors within \pm 30 days of SRS.

\section{Treatment and follow-up}

For all patients, contrast-enhanced T1-weighted MRI of the brain with $1-1.5-\mathrm{mm}$ slice thickness, reconstructed in all three dimensions, was used for delineation of the gross target volume (GTV) and organs at risk. The planning target volume (PTV) was defined as the GTV without any further margin according to common practice for dedicated SRS machines [5]. A stereotactic frame (Leksell G Frame; Elekta AB, Stockholm, Sweden) was used for immobilization for patients treated with the Gamma Knife System (Leksell Gamma Knife 4C). For patient treated with the CyberKnife (Accuray Inc., Sunnyvale, California, USA), thermoplastic masks were used for patient immobilization, and patient localization during treatment was done with stereoscopic radiography image guidance. Additionally, for the CyberKnife, a 1-mm thin slice planning computed tomography with according MRI image registration was generated. Treatment planning was performed using MultiPlan (Accuray Inc.) for the CyberKnife and Leksell Gamma Plan 8.3.1 (Elekta) for the Gamma Knife System according to international best practice guidelines [11]. For the CyberKnife, a mean GTV dose optimization [12] was performed whenever necessary to generate Gamma Knife like plans with high central tumor doses. SRS was applied in a single fraction per metastasis.

Patient follow-up consisted of serial MRI scans (same as for treatment planning) every 8-12 weeks after SRS. Multiple MRIs over a short period of time (if needed every 6-8 weeks) were conducted to diagnose local tumor regrowth. A continuous increase in the size of a lesion (defined as area of contrast enhancement) and contrast uptake in at least two sequential MRI series, nonresponsive to steroids, combined with corresponding perfusion-weighted data was considered as local progression. For differentiation between tumor progression and pseudoprogression, all clinical and radiological data were used. Radiological data in patients with brain metastases routinely included unenhanced T2, FLAIR-, T1-susceptibility-weighted and diffusion-weighted images, ADC-maps, contrast-enhanced T1-weighted images, and perfusion-weighted images, especially CBV-maps. In selected cases with inconclusive routine imaging, $1 \mathrm{H}$ spectroscopic chemical shift imaging was added. Since 2016, all neurooncology patients treated with immunotherapy in our institution are evaluated according to the iRANO (Immunotherapy Response Assessment in Neurooncology) criteria for tumor response. The perfusion-weighted, contrast-enhanced MRI, including the additional images as described before, was the main method for differentiation between progression and pseudoprogression/necrosis.

\section{Statistical analysis}

Follow-up intervals were defined from the date of SRS to the date of the respective event or last contact. Local progression (as defined above) was counted as an event for the endpoint time to local control rate (LCR). Progression of the primary tumor, occurrence of new metastases and local progression were counted as events for the time to progression end point (TTP). Finally, death from any cause was counted as an event for the endpoint overall survival (OS). The Kaplan-Meier method and log-rank test were used for univariate analysis for all time-to-event endpoints. Cox regression and a backwards approach were implemented for multivariate analyses. Pearson's $\chi^{2}$-test was applied to compare categorical data. $P$ values less than or equal to 0.05 were considered significant. All statistics were performed using IBM-SPSS version 25 (IBM Corp., Armonk, New York, USA).

\section{Results}

\section{Patient and tumor characteristics}

Between 1/2012 and 5/2018, a total of 48 patients were treated for 250 brain metastases in 67 SRS treatment sessions. The initial diagnosis of the primary malignancy was between 5/1996 and 2/2018 and the diagnosis of the brain metastases was synchronous in eight (16.7\%) patients and metachronous in $40(83.3 \%)$ patients. The first diagnosis of the brain metastases was between $5 / 2012$ and 4/2018, resulting in a median time to brain metastases after initial diagnosis of 31.7 months (range: 0-216.7 months). The time period between diagnosis of the cerebral metastases and SRS was 1-226 days, with a median of 27.5 days.

Of all patients, 30 (62.5\%) were male and 18 (37.5\%) female. The median age of the patients at the time of SRS was 63 years (range: 30-85 years). Patients presented with a Karnofsky Performance Score (KPS) of $60-100 \%$ with a median of $90 \%$. Additional extracranial 
Table 1 Patient and tumor characteristics

\begin{tabular}{|c|c|}
\hline & Total (\%) \\
\hline Patients & 48 \\
\hline Lesions/patient & 250 \\
\hline Median & 3 \\
\hline \multicolumn{2}{|l|}{ Sex } \\
\hline Male & $30(62.5)$ \\
\hline Female & $18(37.5)$ \\
\hline \multicolumn{2}{|l|}{ Age } \\
\hline Median (range) in years & $63.0(30-85)$ \\
\hline \multicolumn{2}{|l|}{ Karnofsky index } \\
\hline Median (range) in \% & $90(60-100)$ \\
\hline \multicolumn{2}{|l|}{ Extracranial manifestation } \\
\hline No & 16 (33.3) \\
\hline Yes & $32(66.7)$ \\
\hline 1 & $9(18.8)$ \\
\hline 2 & $9(18.8)$ \\
\hline 3 & $3(6.3)$ \\
\hline 4 & $5(10.4)$ \\
\hline 5 & $4(8.3)$ \\
\hline 6 & $2(4.2)$ \\
\hline \multicolumn{2}{|l|}{ Mutational status } \\
\hline BRAF & $23(47.9)$ \\
\hline NRAS & $8(16.7)$ \\
\hline Wild type & $17(35.4)$ \\
\hline \multicolumn{2}{|l|}{ Appearance of metastasis } \\
\hline Metachronous & $40(83.3)$ \\
\hline Synchronous & $8(16.7)$ \\
\hline \multicolumn{2}{|c|}{ Interval between diagnosis of primary tumor and metastasis } \\
\hline Median (range) in months & $31.7(0.0-216.7)$ \\
\hline \multicolumn{2}{|c|}{ Interval between diagnosis of primary tumor and SRS } \\
\hline Median (range) in months & $33.75(0.4-217.2)$ \\
\hline \multicolumn{2}{|c|}{ Interval between diagnosis of metastasis and SRS } \\
\hline Median (range) in days & $27.5(1-226)$ \\
\hline
\end{tabular}

BRAFi, BRAF/MEK inhibitor; NRAS, neuroblastoma rat sarcoma oncogen; SRS, stereotactic radiosurgery.

tumor manifestations were present in $32(66.7 \%)$ of the patients, involving one to six (median 2) extracranial sites at the time of brain SRS. The patient and tumor characteristics are summarized in Table 1.

\section{Radiosurgery characteristics}

Dedicated radiosurgery platforms (Gamma Knife and CyberKnife) were used for all treatments. All lesions were treated with single-fraction SRS. Owing to whole-brain dose constraints, the treatment was regionally split in two fractions separated by four days in two cases (i.e. the metastases in one regional half brain were treated in a single fraction in one session and the other metastases also in a single fraction in a second session). The median total number of radiosurgically treated brain metastases per patient was 3 (range: $1-30$ ). The maximum number of treated metastases in one SRS session was 20, and for a split SRS treatment 21. Of all patients, 34 (71\%) patients received only one SRS session, whereas nine patients had two and five had three sessions owing to distant intracranial recurrence during follow-up. The median PTV per lesion was $0.23 \mathrm{~cm}^{3}$ (range: $0.01-12.27 \mathrm{~cm}^{3}$ ) and the median cumulative PTV was $1.20 \mathrm{~cm}^{3}$ (range: $\left.0.04-28.78 \mathrm{~cm}^{3}\right)$. The median prescribed PTV dose to greater than $98 \%$ tumor coverage was $18.0 \mathrm{~Gy}$ (range: 15-20 Gy), resulting in a median biological equivalent dose with $\alpha / \beta=10 \mathrm{~Gy}\left(\mathrm{BED}_{10}\right)$ of $50.4 \mathrm{~Gy}_{10}$. Prescription was performed on the median $66 \%$ isodose resulting in a maximum dose in each central metastasis of median $28.3 \mathrm{~Gy}$ $\left(\mathrm{BED}_{10}=108.6 \mathrm{~Gy}_{10}\right.$; range: $\left.20.0-36.7 \mathrm{~Gy}\right)$. According to the International Commission on Radiation Units and Measurements (ICRU) report 91 [13] and our national guidelines [14], the PTV $\mathrm{D}_{98 \%}$ and $\mathrm{D}_{2 \%}$ (dose received by 98\% and 2\% of the PTV, respectively) should be reported, though the differences for our cohort were small and details are summarized in Table 2. Twelve (25\%) patients also received WBRT, three of them before and nine of them after SRS.

\section{Targeted therapy and immunotherapy characteristics}

The simultaneously applied systemic therapy was immunotherapy in 45 SRS series, kinase inhibitors in 18 cases and a combination of both in four cases. The kind of simultaneous therapy depended mainly on the mutation state of the primary tumor, which was BRAF mutated in 23 $(47.9 \%)$ patients, neuroblastoma rat sarcoma oncogen mutated in eight $(16.7 \%)$ patients and without known mutation in 17 (35.4\%) patients, and secondly on previous failed therapies. Nivolumab, ipilimumab and pembrolizumab were used as immunotherapy, given every 2-3 weeks, and dabrafenib, trametinib, vemurafenib, cobimetinib and buparlisib as kinase inhibitors, given daily, in our combined patient cohort.

If given before SRS, the last application of the systemic therapy was in median 7.7 days before SRS, and the first application in median 9.2 days after SRS. In 48 of the 67 SRS sessions (71.7\%), patients received their systemic therapy without interruption, delay or dose reduction; 35 patients of these patient received immunotherapy. For the two split session cases, immunotherapy was given in between the 3-day break. In seven (10.8\%) cases the specific systemic therapy was completely stopped before SRS: for three of them the break was due to change of the immunotherapy or targeted therapy agent. In $64.2 \%$ of the treatment series, prescription of dexamethasone for few days after SRS was necessary.

\section{Tumor control and survival}

After a median follow-up of 8.3 months (range: 1.2-43.6 months), the 6-month OS was $75.3 \%$, the 1 -year OS was $50.8 \%$ and the 2-year OS was $31.8 \%$. The progression-free interval after SRS was $42.3 \%$ at 6 months and $25.5 \%$ at 1 year. The LCR was $89.5 \%$ at 1 year (Fig. 1a-c), and all local failures were re-treated with radiosurgery [15].

Despite the small number of events, we conducted exploratory univariate analyses for these three oncological end points. For OS and TTP, we included following factors with possible effect in univariate analyses: sex, age ( $>$ median vs. younger), Karnofsky Performance State, intracerebral progression, mutation state, kind of system therapy and extracranial manifestation. For LCR, we included sex, age, mutation state, kind of system 
Table 2 Treatment characteristics

\begin{tabular}{|c|c|c|}
\hline & Total (\%) & \\
\hline \multicolumn{3}{|l|}{ Treatment series } \\
\hline \multicolumn{3}{|l|}{ Per patient } \\
\hline 1 & $34(70.8)$ & \\
\hline 2 & $9(18.8)$ & \\
\hline 3 & $5(10.4)$ & \\
\hline \multicolumn{3}{|l|}{ Per platform } \\
\hline CyberKnife & $60(89.6)$ & \\
\hline GammaKnife & $7(10.4)$ & \\
\hline \multicolumn{3}{|l|}{ Number of treated lesions } \\
\hline 1 & $23(34.8)$ & \\
\hline 2 & $11(16.7)$ & \\
\hline 3 & $5(7.6)$ & \\
\hline 4 & $8(12.1)$ & \\
\hline 5 & $4(6.1)$ & \\
\hline 6 & $5(7.6)$ & \\
\hline 7 & $2(3.0)$ & \\
\hline 9 & $4(6.1)$ & \\
\hline \multirow{2}{*}{\multicolumn{3}{|c|}{ PTV volume cumulative }} \\
\hline & & \\
\hline Median (range) in $\mathrm{cm}^{3}$ & $1.2(0.039-28.78)$ & \\
\hline \multicolumn{3}{|l|}{ PTV dose } \\
\hline Median (range) in Gy & $18.0(15.0-20.0)$ & \\
\hline \multicolumn{3}{|l|}{ PTV dose BED10 } \\
\hline Median (range) in Gy & $50.4(37.5-60.0)$ & \\
\hline \multicolumn{3}{|l|}{ PTV dose max } \\
\hline Median (range) in Gy & $28.3(20.0-36.7)$ & \\
\hline \multicolumn{3}{|l|}{ PTV dose BED10 max } \\
\hline Median (range) in Gy & $108.6(60.0-171.4)$ & \\
\hline \multicolumn{3}{|l|}{ PTV dose D98\% } \\
\hline \multirow{2}{*}{\multicolumn{3}{|c|}{$\begin{array}{l}\text { Median (range) in Gy } \\
\text { PTV dose BED10 D98\% }\end{array}$}} \\
\hline & & \\
\hline Median (range) in Gy & $51.2(38.1-61.3)$ & \\
\hline \multicolumn{3}{|l|}{ PTV dose D2\% } \\
\hline Median (range) in Gy & $27.8(19.6-36.0)$ & \\
\hline \multicolumn{3}{|l|}{ PTV dose BED10 D2\% } \\
\hline Median (range) in Gy & $104.9(58.0-165.3)$ & \\
\hline \multicolumn{3}{|l|}{ Whole-brain radiotherapy } \\
\hline Total & $12(25.1)$ & \\
\hline Before SRS & $3(6.3)$ & \\
\hline After SRS & $9(18.8)$ & \\
\hline \multicolumn{3}{|l|}{ Systemic therapy (SRS series) } \\
\hline Immunotherapy & $45(67.1)$ & \\
\hline PD-1/PD-L1 & $22(32.8)$ & \\
\hline CTLA-4 & $14(20.9)$ & \\
\hline PD-1/CTLA-4 & $9(13.4)$ & \\
\hline Kinase inhibitor & $18(26.9)$ & \\
\hline $\mathrm{ICI}+\mathrm{BRAFi}$ & $4(6.0)$ & \\
\hline \multicolumn{3}{|l|}{ Dexamethasone during SRS } \\
\hline No & $24(35.8)$ & \\
\hline Yes & $43(64.2)$ & \\
\hline Median (range) (mg) & $4(0-8)$ & \\
\hline Timing of SRS and systemic therapy & $\mathrm{ICl}$ (in \%) & BRAFi (in \%) \\
\hline Before/concomitant to SRS & $40(61.5)$ & $15(23.1)$ \\
\hline Start after SRS & $7(10.8)$ & $3(4.6)$ \\
\hline
\end{tabular}

BED, biological equivalent dose; BRAFi, BRAF/MEK-inhibitor; ICI, immune checkpoint inhibitor; PTV, planning target volume; SRS, stereotactic radiosurgery.

therapy, PTV volume and PTV dose with several equivalents.

Regarding OS, the application of immunotherapy significantly correlated with a better outcome $(P=0.031$, Fig. 2a). This was also the case for an application of systemic therapies directly before or concomitant to SRS when compared with cases with initiation of systemic therapy shortly after SRS $(P=0.039$, Fig. 2b). These two parameters remained significant in multivariate analysis $(P=0.037$ and 0.045 , respectively). In the subgroup of patients treated
Fig. 1
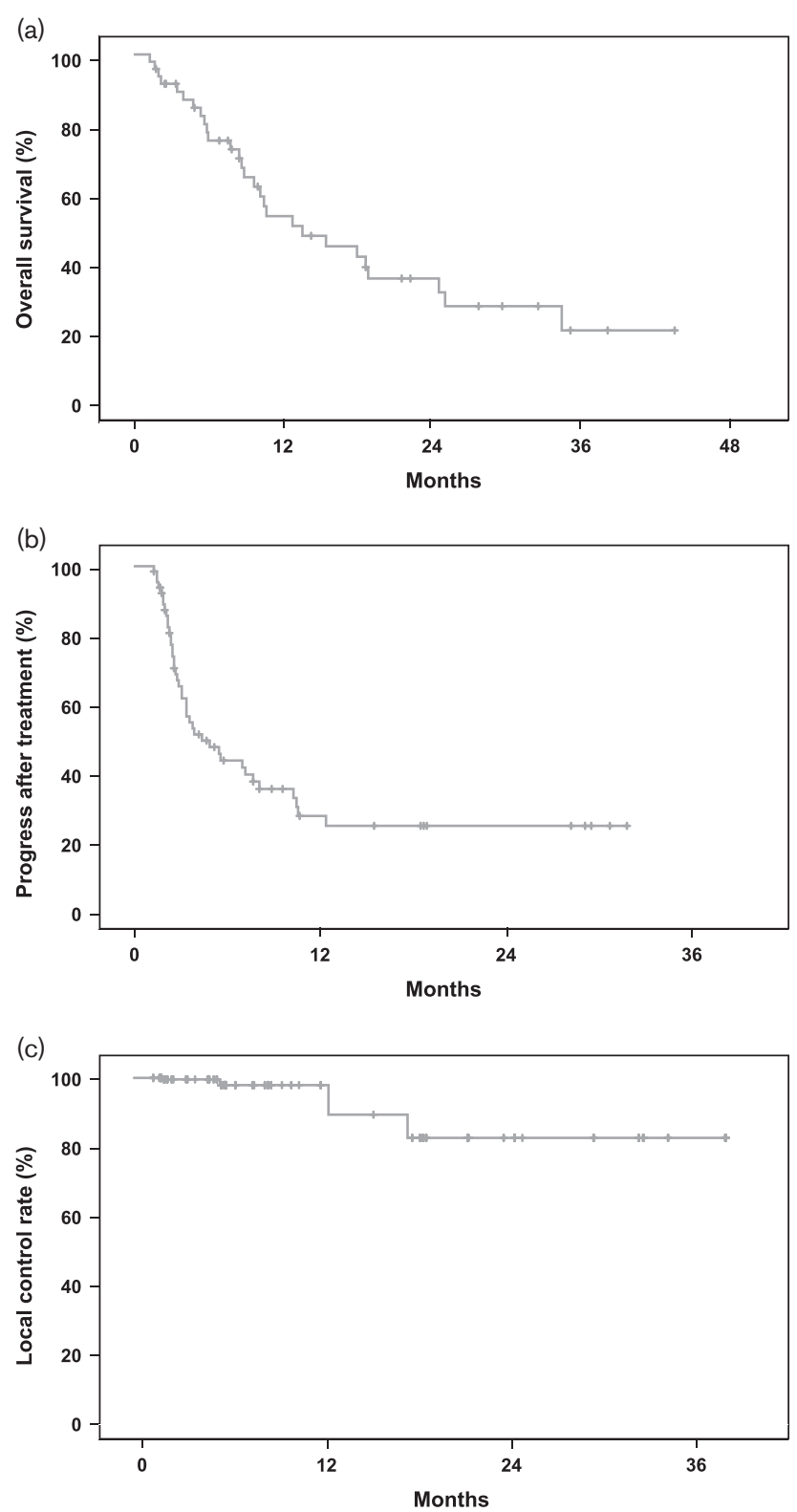

Oncological outcome. BRAFi, BRAF/MEK inhibitor; ICl, immune checkpoint inhibitor; SRS, stereotactic radiosurgery.

with ICI, PD-1-inhibitors alone or as combination-partners were associated with improved survival in univariate analysis compared with anti-CTLA-4 treatment $(P=0.038$, Fig. 3). When examining TTP, the sequence of application (before/concomitant to vs. after SRS) was the only significant factor associated with outcome $(P=0.027$, Fig. 2c), whereas ICI medication showed only a trend for better results compared with kinase inhibitors $(P=0.112)$. For LCR, we could not identify any factors with positive or negative effect. Neither age, sex, Karnofsky performance status, number of metastases nor extracranial involvement sites proved to have 
Fig. 2
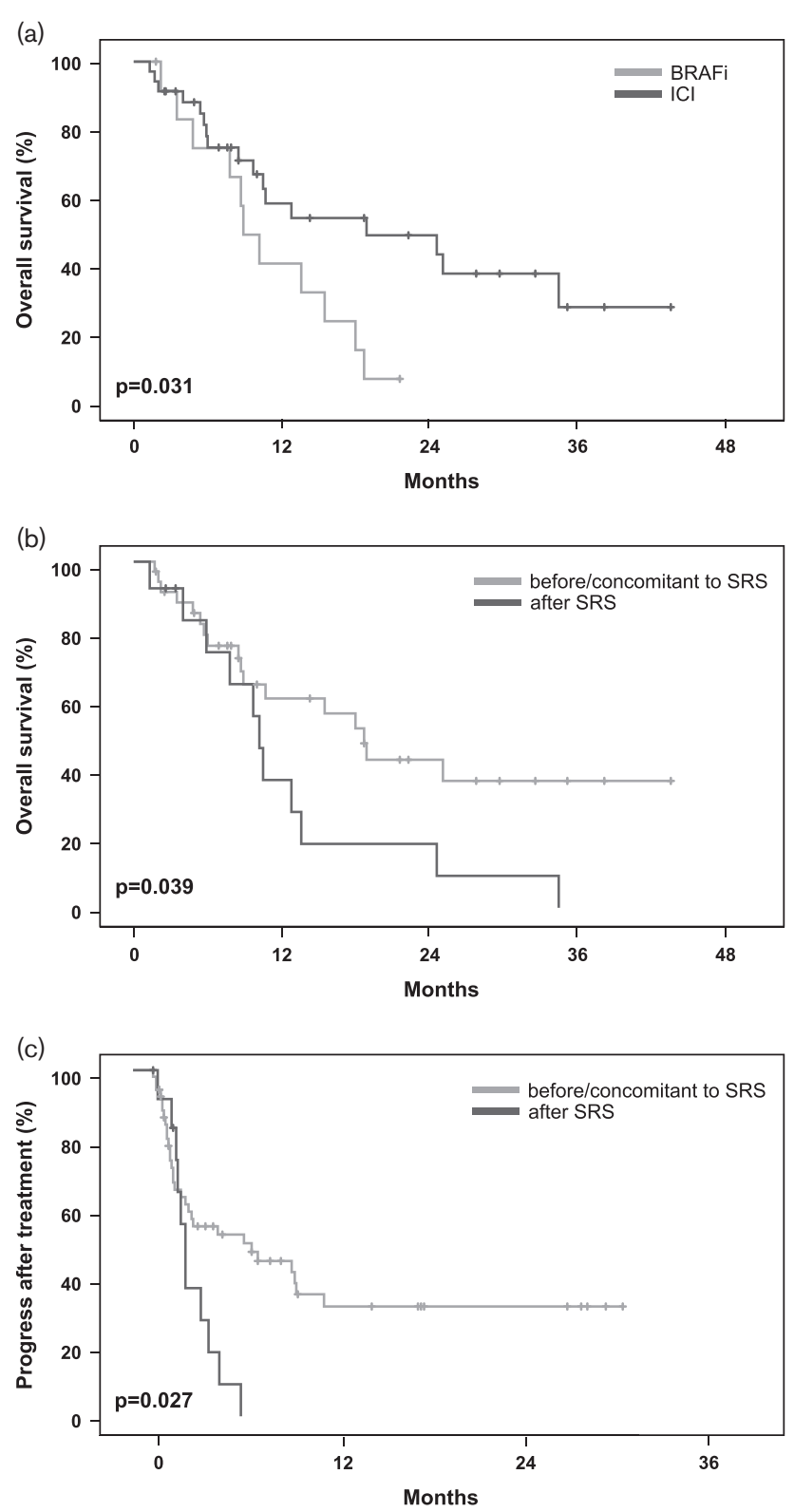

Oncological outcome depending on kind of and timing of systemic therapies. BRAFi, BRAF/MEK inhibitor; ICl, immune checkpoint inhibitor; SRS, stereotactic radiosurgery.

any effect on tumor control or survival. These results are presented in Table 3 .

\section{Toxicity}

Of 48 patients, 34 (70.8\%) did not experience any toxicity, either SRS related or systemic treatment related. Three patients experienced altogether four grade III toxicities, attributable to both the SRS and concomitant systemic therapy, namely, one patient with autoimmune pancreatitis, one patient with autoimmune hypophysitis and one patient with two cases of symptomatic radio-necrosis, with
Fig. 3

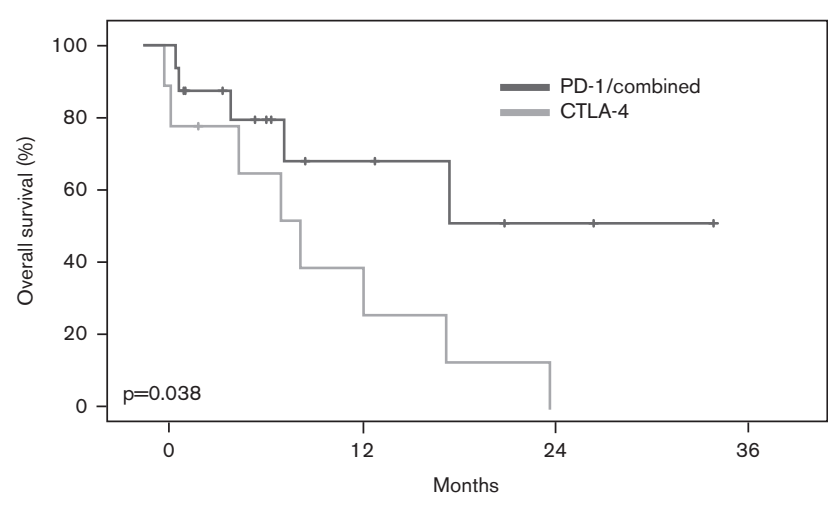

Oncological outcome depending on immunotherapy agents. PD-1, antiprogrammed cell death protein 1 ; CTLA-4, anti-cytotoxic T-lymphocyteassociated protein 4 .

symptoms that resolved after surgery. The other eleven patients with adverse effects after SRS showed subclinical radionecrosis, intracerebral bleeding, headache or other mild neurological symptoms, which may be attributed to SRS, such as nausea. Diarrhea, leucopenia, autoimmune hepatitis, and autoimmune pancreatitis were also observed and are most likely attributed to the systemic therapy. One patient experienced a seizure, and the differentiation between treatment-toxicity and tumor progression was inconclusive.

Of the four patients with radionecrosis, only one had WBRT. The patient with the grade III radionecrosis (operated) had received three series of SRS for a total of 30 metastases with regionally overlapping treatment fields. An overview of all adverse events and the corresponding systemic treatments can be found in Table 4 .

\section{Discussion}

Despite the fact that both SRS and targeted/immunetherapies are considered standard treatment options for brain metastases of malignant melanoma, the optimal combination strategy and sequence of these modalities remains largely unknown $[3,4,7,16,17]$. The implementation of BRAF/MEKi and the advent of immunotherapy revolutionized treatment of metastatic melanoma compared with the unsatisfactory control rates provided with classical chemotherapy [18]. Combining such agents with radiotherapy opens new promising options for synergistic effects but also raises safety and feasibility concerns as new and unexpected toxicities may arise [19].

In this context, SRS appears to be the ideal irradiation technique for this indication, as it provides maximal sparing of healthy tissue, and treatment duration is much shorter as in the case of fractionated radiotherapy, allowing for only very short interruptions of systemic treatments where necessary. Besides, although novel drugs provide not only 


\begin{tabular}{|c|c|c|c|}
\hline & Overall survival & Time to progression & Local control \\
\hline \multicolumn{4}{|l|}{ Univariate analysis ( $P$ value) } \\
\hline Age (>63 vs. $\leq 63$ years) & 0.591 & 0.313 & 0.582 \\
\hline ECOG Index (0 vs. 1) & 0.547 & 0.347 & \\
\hline Sex (male vs. female) & 0.418 & 0.290 & 0.882 \\
\hline Intracerebral progression & 0.519 & 0.000 & \\
\hline Mutational status (BRAF/NRAS vs. wild) & 0.289 & 0.117 & 0.749 \\
\hline Mutational status (BRAF vs. NRAS) & 0.370 & 0.939 & 0.458 \\
\hline Mutational status (BRAF vs. wild type) & 0.751 & 0.219 & 0.560 \\
\hline Mutational status (NRAS vs. wild type) & 0.295 & 0.662 & 0.542 \\
\hline Immunotherapy vs. kinase inhibitor & 0.031 & 0.112 & 0.754 \\
\hline Immunotherapy agents (anti-PD-1 monotherapy and combinations vs. anti-CTLA-4) & 0.038 & 0.169 & 0.195 \\
\hline Application of systemic therapies (directly before/concomitant vs. after SRS) & 0.039 & 0.027 & 0.206 \\
\hline Extracranial manifestation (no vs. yes) & 0.107 & 0.684 & \\
\hline Extracranial manifestation ( 1 vs. $\geq 2$ ) & 0.201 & 0.671 & \\
\hline Extracranial manifestation ( 2 vs. $\geq 3$ ) & 0.065 & 0.792 & \\
\hline Extracranial manifestation ( 3 vs. $\geq 4$ ) & 0.143 & 0.792 & \\
\hline Number of metastases ( 3 vs. $\geq 4)$ & 0.471 & 0.863 & \\
\hline Number of metastases ( 4 vs. $\geq 5$ ) & 0.471 & 0.863 & \\
\hline Number of metastases $(5$ vs. $\geq 6)$ & 0.471 & 0.863 & \\
\hline Number of metastases ( $10 \mathrm{vs} . \geq 11)$ & 0.471 & 0.863 & \\
\hline PTV volume $\left(>0.225\right.$ vs. $\left.\leq 0.225 \mathrm{~cm}^{3}\right)$ & & & 0.296 \\
\hline PTV volume all (>1.2 vs. $\left.\leq 1.2 \mathrm{~cm}^{3}\right)$ & & & 0.894 \\
\hline PTV dose (>18.0 vs. $\leq 18.0$ Gy) & & & 0.408 \\
\hline Maximum PTV dose (>28.3 vs. $\leq 28.3$ Gy) & & & 0.353 \\
\hline PTV D98 (>18.2 vs. $\leq 18.2$ Gy) & & & 0.376 \\
\hline PTV D98 BED 10 (>51.2 Gy vs. $\leq 51.2 \mathrm{~Gy})$ & & & 0.376 \\
\hline PTV D2 (>27.8 Gy vs. $\leq 27.8$ Gy) & & & 0.400 \\
\hline PTV D2 BED $_{10}(>104.9$ Gy vs. $\leq 104.9$ Gy) & & & 0.400 \\
\hline WBRT (after SRS vs. no) & 0.116 & 0.745 & 0.593 \\
\hline \multicolumn{4}{|l|}{ Multivariate analysis ( $P$ value) } \\
\hline Immunotherapy vs. kinase inhibitor & 0.037 & & \\
\hline Timing of systemic therapies (directly before/concomitant vs. after SRS) & 0.045 & & \\
\hline Immunotherapy agents (anti-PD-1 monotherapy and combinations vs. anti-CTLA- 4$)^{\mathrm{a}}$ & 0.142 & & \\
\hline
\end{tabular}

BED, biological equivalent dose; CTLA-4, anti-cytotoxic T-lymphocyte-associated protein 4; anti-PD-1, anti-programmed cell death protein 1; NRAS, neuroblastoma rat sarcoma oncogen; PTV, planning target volume; SRS, stereotactic radiosurgery; WBRT, whole-brain radiotherapy.

a Only patients with immunotherapy and the variables 'timing' and 'immunotherapy agents' included for this analysis.

Bold values indicate significance $(P<0.05)$.

improved systemic but also local control [17], recent efforts to omit, or at least defer, radiotherapy in patients with brain metastases, using only upfront targeted therapies, resulted in significantly inferior survival in patients with non-small cellular lung cancer [20]. On the contrary, there exist preclinical and clinical data and a strong biological rationale for combining both BRAF-inhibitors or ICI with radiotherapy: Samabade et al. [21] could demonstrate that many radioresistant melanoma cell lines were BRAF mutated, so that BRAF inhibition enhanced radiosensitivity. Dasgupta $e t$ al. [22] showed that the simultaneous BRAF inhibition and irradiation led to more apoptotic tumor cells and prolonged survival of mouse models. In a clinical study, blood derived from patients with melanoma treated with vemurafenib also showed increased sensitivity to radiation [19]. Ionizing radiation can also facilitate immune response through various mechanisms like secretion of danger signals (e.g. HMGB-1 and calreticulin) and increased antigen presentation, potentially improving response to anti-CTLA-4 and anti-PD-1 treatment [23-25]. However, the necessary minimum and maximum radiation doses and the fractionation schemes for enabling such effects are still widely under investigation. Strong evidence, at least for immunotherapy, suggests that higher radiosurgery doses are required [26].
In the present study, we examined and compared the aforementioned combinations (SRS + BRAF/MEKi and SRS + ICI) regarding feasibility/toxicity and oncological results in a cohort of 48 patients with 250 brain metastases treated with SRS dedicated platforms (Gamma Knife and CyberKnife with Gamma Knife like planning, i.e. very inhomogeneous dose distribution inside the central metastases). Although there exist some recent data about toxicity and efficacy of similar regimens, all of these series are retrospective, mostly included smaller patient numbers, or investigated solely and separately the combination of SRS with BRAF/MEKi [10,27-29] or with ICI [30-33]. Furthermore, only a limited number of metastases were treated with each SRS session in those studies. We could identify only two larger series including SRS combined with all possible approved drugs for metastatic melanoma: Ahmed et al. [34] reported on 96 patients treated for 314 lesions, but 20 cases with chemotherapy were also included among them. Furthermore, the patients were all treated at a linear accelerator, the median number of irradiated lesions per patient (with the exception of the chemotherapy cases) was 2 and the median GTV amounted $0.14 \mathrm{~cm}^{3}$, whereas in our series, the median number of treated lesions per patient was higher $(n=3)$ and the median GTV was 
Table 4 Toxicity

\begin{tabular}{|c|c|c|c|}
\hline Adverse effect & System therapy & $\begin{array}{l}\text { Grade I/II } \\
{[n(\%)]}\end{array}$ & $\begin{array}{l}\text { Grade III } \\
\text { [n (\%)] }\end{array}$ \\
\hline$\%$ of all treated lesions & $n=250$ & & \\
\hline \multicolumn{4}{|l|}{ Radionecrosis } \\
\hline & Ipilimumab & $1(0.4)$ & \\
\hline & $\begin{array}{l}\text { Nivolumab/ } \\
\text { Ipilimumab }\end{array}$ & $1(0.4)$ & $1(0.4)$ \\
\hline & Pembrolizumab & & \\
\hline & Vemurafenib & $5(2.0)$ & \\
\hline & Dabrafenib & $1(0.4)$ & \\
\hline & $\begin{array}{l}\text { Dabrafenib/ } \\
\text { Trametinib }\end{array}$ & $1(0.4)$ & $1(0.4)$ \\
\hline \multicolumn{4}{|l|}{ Intracerebral bleeding } \\
\hline & Ipilimumab & $1(0.4)$ & \\
\hline & Pembrolizumab & $2(0.8)$ & \\
\hline & Dabrafenib & $1(0.4)$ & \\
\hline$\%$ of all SRS series & $n=67$ & & \\
\hline \multicolumn{4}{|l|}{ Headache } \\
\hline & $\begin{array}{l}\text { Nivolumab/ } \\
\text { Ipilimumab }\end{array}$ & $1(1.5)$ & \\
\hline & Pembrolizumab & $1(1.5)$ & \\
\hline \multicolumn{4}{|l|}{ Nausea } \\
\hline & $\begin{array}{l}\text { Nivolumab/ } \\
\text { Ipilimumab }\end{array}$ & $1(1.5)$ & \\
\hline & Pembrolizumab & $1(1.5)$ & \\
\hline \multicolumn{4}{|l|}{ Leucopenia } \\
\hline & Vemurafenib & $1(1.5)$ & \\
\hline \multicolumn{4}{|l|}{ Diarrhea } \\
\hline & $\begin{array}{l}\text { Nivolumab/ } \\
\text { Ipilimumab }\end{array}$ & $1(1.5)$ & \\
\hline \multicolumn{4}{|l|}{ Seizure } \\
\hline & $\begin{array}{l}\text { Nivolumab/ } \\
\text { Ipilimumab }\end{array}$ & $1(1.5)$ & \\
\hline \multicolumn{4}{|l|}{$\begin{array}{l}\text { Other neurological } \\
\text { symptoms }\end{array}$} \\
\hline & Ipilimumab & $1(1.5)$ & \\
\hline & $\begin{array}{l}\text { Nivolumab/ } \\
\text { Ipilimumab }\end{array}$ & $1(1.5)$ & \\
\hline & Pembrolizumab & $1(1.5)$ & \\
\hline & Vemurafenib & $1(1.5)$ & \\
\hline \multicolumn{4}{|l|}{ Autoimmune hepatitis } \\
\hline & Ipilimumab & $1(1.5)$ & \\
\hline & Pembrolizumab & $1(1.5)$ & \\
\hline \multicolumn{4}{|l|}{$\begin{array}{l}\text { Autoimmune } \\
\text { pancreatitis }\end{array}$} \\
\hline & $\begin{array}{l}\text { Nivolumab/ } \\
\text { Ipilimumab }\end{array}$ & & $1(1.5)$ \\
\hline \multicolumn{4}{|l|}{$\begin{array}{l}\text { Autoimmune } \\
\text { hypophysitis }\end{array}$} \\
\hline & Pembrolizumab & & $1(1.5)$ \\
\hline
\end{tabular}

SRS, stereotactic radiosurgery.

somewhat larger $\left(0.23 \mathrm{~cm}^{3}\right)$. Despite these differences in treated targets and platform (and correlated dose prescription), the median OS after SRS for all patients was in both studies between 8 and 9 months, and the 1-year LCRs, with $89.5 \%$ (our data) and $83 \%$, surprisingly similar. Moreover, in both analyses, there was no factor with a significant effect on local control, and the only type of systemic therapy that affected OS was immunotherapy, which was generally associated with improved survival compared with BRAF/MEKi treatments. In an even larger study, Kotecha et al. [35] analyzed 366 patients with 793 SRS-treated lesions (median number per patient 2$)$. Only $15 \%(n=54)$ of the patients received ICI or BRAF/MEKi; the interval between SRS and systemic therapy was very heterogeneous, and no exact volume calculation was reported. The BRAF status had not been available for all patients, but BRAF inhibition within 30 days of SRS was associated with lower local failure rates. Interestingly, ICI seemed to be associated with local only in BRAF-mutated cases. The 1-year OS in the BRAF-positive subgroup reached 53\% and LCR for the entire cohort was $85.0 \%$, which is in line with our data (51.0 and $89.5 \%$, respectively). In a multivariate analysis, both BRAF/MEKi and ICI correlated with improved survival, but only BRAF/MEKi with LCR. There was no direct comparison between the two different treatment groups, and the heterogeneous inclusion criteria (e.g. patients after surgery, or with unknown BRAFstatus were included) make interpretation of the data challenging.

Mounting evidence suggests an enhanced effect of concomitant application of SRS together with BRAF/MEKi [35] and ICI [31-33]. Yet, it remains to be elucidated how narrow the timeframe of the combination should be and if drug application shortly before or after SRS could provide comparable results. In the present study, we could also show an association between timing and outcome, with a concomitant setting appearing superior in terms of OS and TTP. However, as the LCR did differ depending on the sequence of SRS and targeted treatment, this prolonged survival may be attributed to improved systemic control. It remains unclear, if this improved systemic control can be associated with SRS, for example, through immunological and/or abscopal effects, or with cohortrelated bias. Nevertheless, the 1-year OS (50.8\%) and 1-year LCR $(89.5 \%)$ were similar or even better compared with historical series of SRS alone, although the median and maximum number of metastases in our cohort was higher ( $n=3$ and 20, respectively) [36-38]. Intriguingly, clinical parameters with known effect on outcome, like previous WBRT, number of cerebral or extracranial metastases and Karnofsky status [39] did not seem to play a crucial role in this cohort. Notwithstanding the limitations of this retrospective analysis, a highly effective local treatment, like SRS, combined with modern, active systemic therapy may successfully control even advanced disease stages. Preclinical data seem to confirm a synergistic effect for immunotherapy and SRS, which might also explain the higher survival rates observed in our series [40].

The last exploratory survival analysis conducted was a comparison of the different immunotherapy regimens in the respective subgroup treated with ICI. Patients receiving anti-PD1 treatment had a clear survival benefit compared with those treated with anti-CTLA-4 monotherapy in the univariate analysis, without any significant difference in local control or progression-free survival. The improved OS after anti-PD1 treatment (monotherapy or combined with anti-CTLA-4) is in accordance with the large prospective trials for metastasized melanoma [41] and such not surprising. Although the similar 
local control for both groups could easily be explained through the added effect of radiosurgery, the lack of significance in terms of time to progression or OS in multivariate analysis is rather associated with the very limited number of patients and events for these two small sub-cohorts of the ICI-subgroup.

Regarding toxicity, we could only identify four grade III adverse events of which only two were related to SRS: two cases of clinically apparent radio-necrosis associated with a status epilepticus, requiring surgical, one after combined treatment with ICI and one with BRAFi, both in the same patient, but after different SRS sessions. This relatively low rate is in accordance with the literature reporting grade III toxicity in general less than $10 \%$, whereas series examining only CTLA-4 inhibitors reported higher rates of radionecrosis of about 20\% [30,33]. Note that these more toxic agents were underrepresented in our cohort. Recent comprehensive reviews on this topic confirm our observations [6,42]. Moreover, there was no statistically significant difference in the steroid requirement between the different patient groups. Some authors associate simultaneous application of vemurafenib and radiotherapy with increased toxicity $[10,35]$. However, WBRT and (resulting) skin toxicity were also included in these studies and the overall rates of severe adverse effects remain low. No unexpected or life-threatening toxicity occurred, and all ICI-associated or BRAF/MEKi-associated adverse effects were manageable and resolved.

This study has several limitations: the limited number of patients and lesions, the heterogeneity of treatment regimens used, and the retrospective nature of the analysis even though our databases are prospectively generated under strict institutional protocols. Furthermore, the tumor volumes were generally small in our cohort and we applied only single fraction doses. For larger metastases, a fractionated approach may be necessary [43]. Despite these limitations, this study, reporting of 250 metastases, is one of the largest so far and one of the very few including patients treated with both kinase inhibitors and ICI in various combinations.

Taken together, the combination of SRS with novel targeted agents is a feasible option with an acceptable safety profile and good clinical outcome even for treating a higher number of metastases and could be used to defer WBRT.

\section{Acknowledgements Conflicts of interest}

There are no conflicts of interest.

\section{References}

1 Sloan AE, Nock CJ, Einstein DB. Diagnosis and treatment of melanoma brain metastasis: a literature review. Cancer Control 2009; 16:248-255.

2 Dummer R, Hauschild A, Lindenblatt N, Pentheroudakis G, Keilholz U, Committee EG. Cutaneous melanoma: ESMO Clinical Practice Guidelines for diagnosis, treatment and follow-up. Ann Oncol 2015; 26 (Suppl 5):v126-v132.
3 Glitza Oliva IC, Schvartsman G, Tawbi H. Advances in the systemic treatment of melanoma brain metastases. Ann Oncol 2018; 29:1509-1520.

4 Goyal S, Silk AW, Tian S, Mehnert J, Danish S, Ranjan S, et al. Clinical management of multiple melanoma brain metastases: a systematic review. JAMA Oncol 2015; 1:668-676.

5 Kocher M, Wittig A, Piroth MD, Treuer H, Seegenschmiedt H, Ruge M, et al. Stereotactic radiosurgery for treatment of brain metastases. A report of the DEGRO Working Group on Stereotactic Radiotherapy. Strahlenther Onkol 2014; 190:521-532.

6 Kroeze SG, Fritz C, Hoyer M, Lo SS, Ricardi U, Sahgal A, et al. Toxicity of concurrent stereotactic radiotherapy and targeted therapy or immunotherapy: a systematic review. Cancer Treat Rev 2017; 53:25-37.

7 Gabani P, Fischer-Valuck BW, Johanns TM, Hernandez-Aya LF, Keller JW, Rich $\mathrm{KM}$, et al. Stereotactic radiosurgery and immunotherapy in melanoma brain metastases: patterns of care and treatment outcomes. Radiother Oncol 2018; 128: 266-273.

8 Diao K, Bian SX, Routman DM, Yu C, Ye JC, Wagle NA, et al. Stereotactic radiosurgery and ipilimumab for patients with melanoma brain metastases: clinical outcomes and toxicity. J Neurooncol 2018; 139:421-429.

9 Rahman R, Cortes A, Niemierko A, Oh KS, Flaherty KT, Lawrence DP, et al The impact of timing of immunotherapy with cranial irradiation in melanoma patients with brain metastases: intracranial progression, survival and toxicity. J Neurooncol 2018; 138:299-306.

10 Hecht M, Meier F, Zimmer L, Polat B, Loquai C, Weishaupt C, et al. Clinical outcome of concomitant vs interrupted BRAF inhibitor therapy during radiotherapy in melanoma patients. $\mathrm{Br} J$ Cancer 2018; 118:785-792.

11 Moustakis C, Chan MKH, Kim J, Nilsson J, Bergman A, Bichay TJ, et al. Treatment planning for spinal radiosurgery: a competitive multiplatform benchmark challenge. Strahlenther Onkol 2018; 194:843-854.

12 Stera S, Balermpas P, Chan MKH, Huttenlocher S, Wurster S, Keller C, et al. Breathing-motion-compensated robotic guided stereotactic body radiation therapy: patterns of failure analysis. Strahlenther Onkol 2018; 194:143-155.

13 Schauer A. Prescribing, recording, and reporting of stereotactic treatments with small photon beams. J ICRU 2014; 14:1-160.

14 Wilke LAN, Blanck O, Brunner TB, Combs S, Grosu AL, Moustakis C, et al. ICRU report 91 on prescribing, recording, and reporting of stereotactic treatments with small photon beams - statement from the DEGRO/DGMP Working Group Stereotactic Radiotherapy and Radiosurgery. Strahlenther Onkol 2018; 190:521-532.

15 Balermpas P, Stera S, Muller von der Grun J, Loutfi-Krauss B, Forster MT, Wagner $\mathrm{M}$, et al. Repeated in-field radiosurgery for locally recurrent brain metastases: feasibility, results and survival in a heavily treated patient cohort. PLoS One 2018; 13:e0198692.

16 Goldberg SB, Gettinger SN, Mahajan A, Chiang AC, Herbst RS, Sznol M, et al. Pembrolizumab for patients with melanoma or non-small-cell lung cancer and untreated brain metastases: early analysis of a non-randomised, open-label, phase 2 trial. Lancet Oncol 2016; 17:976-983.

17 Long GV, Atkinson V, Lo S, Sandhu S, Guminski AD, Brown MP, et al. Combination nivolumab and ipilimumab or nivolumab alone in melanoma brain metastases: a multicentre randomised phase 2 study. Lancet Oncol 2018; 19:672-681

18 Agarwala SS, Kirkwood JM, Gore M, Dreno B, Thatcher N, Czarnetski B, et al. Temozolomide for the treatment of brain metastases associated with metastatic melanoma: a phase II study. J Clin Oncol 2004; 22:2101-2107.

19 Hecht M, Zimmer L, Loquai C, Weishaupt C, Gutzmer R, Schuster B, et al. Radiosensitization by BRAF inhibitor therapy-mechanism and frequency of toxicity in melanoma patients. Ann Oncol 2015; 26:1238-1244.

20 Magnuson WJ, Lester-Coll NH, Wu AJ, Yang TJ, Lockney NA, Gerber NK, et al. Management of brain metastases in tyrosine kinase inhibitor-naive epidermal growth factor receptor-mutant non-small-cell lung cancer: a retrospective multi-institutional analysis. J Clin Oncol 2017; 35:1070-1077.

21 Sambade MJ, Peters EC, Thomas NE, Kaufmann WK, Kimple RJ, Shields JM. Melanoma cells show a heterogeneous range of sensitivity to ionizing radiation and are radiosensitized by inhibition of B-RAF with PLX-4032. Radiother Oncol 2011; 98:394-399.

22 Dasgupta T, Olow AK, Yang X, Hashizume R, Nicolaides TP, Tom M, et al. Survival advantage combining a BRAF inhibitor and radiation in BRAF V600E-mutant glioma. J Neurooncol 2016; 126:385-393.

23 Sharabi AB, Nirschl CJ, Kochel CM, Nirschl TR, Francica BJ, Velarde E, et al. Stereotactic radiation therapy augments antigen-specific PD-1-mediated antitumor immune responses via cross-presentation of tumor antigen. Cancer Immunol Res 2015; 3:345-355.

24 Pilones KA, Vanpouille-Box C, Demaria S. Combination of radiotherapy and immune checkpoint inhibitors. Semin Radiat Oncol 2015; 25:28-33. 
25 Vanpouille-Box C, Pilones KA, Wennerberg E, Formenti SC, Demaria S. In situ vaccination by radiotherapy to improve responses to anti-CTLA-4 treatment. Vaccine 2015; 33:7415-7422.

26 Giordano FA, Veldwijk MR, Herskind C, Wenz F. Radiotherapy, tumor mutational burden, and immune checkpoint inhibitors: time to do the math. Strahlenther Onkol 2018; 194:873-875.

27 Chowdhary M, Patel KR, Danish HH, Lawson DH, Khan MK. BRAF inhibitors and radiotherapy for melanoma brain metastases: potential advantages and disadvantages of combination therapy. Onco Targets Ther 2016; 9:7149-7159.

28 Gaudy-Marqueste C, Carron R, Delsanti C, Loundou A, Monestier S, Archier $\mathrm{E}$, et al. On demand Gamma-Knife strategy can be safely combined with BRAF inhibitors for the treatment of melanoma brain metastases. Ann Oncol 2014; 25:2086-2091.

29 Ahmed KA, Freilich JM, Sloot S, Figura N, Gibney GT, Weber JS, et al. LINAC-based stereotactic radiosurgery to the brain with concurrent vemurafenib for melanoma metastases. J Neurooncol 2015; 122:121-126.

30 Skrepnik T, Sundararajan S, Cui H, Stea B. Improved time to disease progression in the brain in patients with melanoma brain metastases treated with concurrent delivery of radiosurgery and ipilimumab. Oncoimmunology 2017; 6:e1283461.

31 Cohen-Inbar O, Shih HH, Xu Z, Schlesinger D, Sheehan JP. The effect of timing of stereotactic radiosurgery treatment of melanoma brain metastases treated with ipilimumab. J Neurosurg 2017; 127:1007-1014.

32 Yusuf MB, Amsbaugh MJ, Burton E, Chesney J, Woo S. Peri-SRS administration of immune checkpoint therapy for melanoma metastatic to the brain: investigating efficacy and the effects of relative treatment timing on lesion response. World Neurosurg 2017; 100:632-640 e634.

33 Kiess AP, Wolchok JD, Barker CA, Postow MA, Tabar V, Huse JT, et al. Stereotactic radiosurgery for melanoma brain metastases in patients receiving ipilimumab: safety profile and efficacy of combined treatment. Int J Radiat Oncol Biol Phys 2015; 92:368-375.
34 Ahmed KA, Stallworth DG, Kim Y, Johnstone PA, Harrison LB, Caudell JJ, et al. Clinical outcomes of melanoma brain metastases treated with stereotactic radiation and anti-PD-1 therapy. Ann Onco/ 2016; 27:434-441.

35 Kotecha R, Miller JA, Venur VA, Mohammadi AM, Chao ST, Suh JH, et al. Melanoma brain metastasis: the impact of stereotactic radiosurgery, BRAF mutational status, and targeted and/or immune-based therapies on treatment outcome. J Neurosurg 2018; 129:50-59.

36 Hara W, Tran P, Li G, Su Z, Puataweepong P, Adler JR Jr, et al. Cyberknife for brain metastases of malignant melanoma and renal cell carcinoma. Neurosurgery 2009; 64 (2 Suppl):A26-A32.

37 Liew DN, Kano H, Kondziolka D, Mathieu D, Niranjan A, Flickinger JC, et al. Outcome predictors of Gamma Knife surgery for melanoma brain metastases. Clinical article. J Neurosurg 2011; 114:769-779.

38 Lwu S, Goetz P, Monsalves E, Aryaee M, Ebinu J, Laperriere N, et al. Stereotactic radiosurgery for the treatment of melanoma and renal cell carcinoma brain metastases. Oncol Rep 2013; 29:407-412.

39 Huttenlocher S, Sehmisch L, Schild SE, Blank O, Hornung D, Rades D. Identifying melanoma patients with 1-3 brain metastases who may benefit from whole-brain irradiation in addition to radiosurgery. Anticancer Res 2014; 34:5589-5592.

40 Vanpouille-Box C, Alard A, Aryankalayil MJ, Sarfraz Y, Diamond JM, Schneider RJ, et al. DNA exonuclease Trex1 regulates radiotherapy-induced tumour immunogenicity. Nat Commun 2017; 8:15618.

41 Wolchok JD, Chiarion-Sileni V, Gonzalez R, Rutkowski P, Grob JJ, Cowey $\mathrm{CL}$, et al. Overall survival with combined nivolumab and ipilimumab in advanced melanoma. N Engl J Med 2017; 377:1345-1356.

42 Hwang WL, Pike LRG, Royce TJ, Mahal BA, Loeffler JS. Safety of combining radiotherapy with immune-checkpoint inhibition. Nat Rev Clin Oncol 2018; 15:477-494.

43 Zindler JD, Schiffelers J, Lambin P, Hoffmann AL. Improved effectiveness of stereotactic radiosurgery in large brain metastases by individualized isotoxic dose prescription: an in silico study. Strahlenther Onkol 2018; 194:560-569. 